\title{
Recent treatment advances in HER2-positive metastatic breast cancer: a clinical approach
}

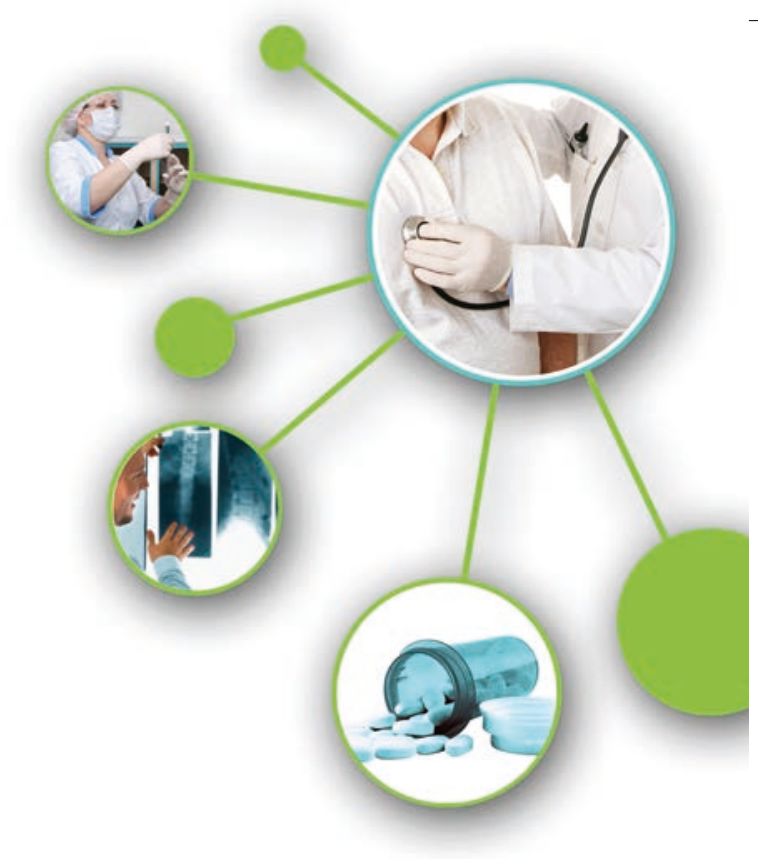

\section{Practice Points}

- Treatment decision in HER2-positive metastatic breast cancer patients must be based on patient factors including evaluation of extent of disease, assessment of performance status, review of cardiac status and consideration of previous treatment including adjuvant taxanes and trastuzumab.

- Generally, taxanes, along with trastuzumab, remain the standard first-line approach. However, recent evidence suggests that patients may be considered for vinorelbine and trastuzumab based on superior toxicity profile and possible improved efficacy.

- Pertuzumab, along with docetaxel and trastuzumab, has demonstrated improved progression-free survival and may be the new standard therapy; however, appropriate cost-effectiveness studies need to be conducted.

- HER2- and hormone receptor-positive metastatic breast cancer patients with low-burden visceral disease and a prolonged disease-free interval may be candidates for treatment with either anastrozole and trastuzumab or lapatinib and letrozole.

- There is a need for prospective studies and predictive biomarkers to determine which patients could be treated with anti-HER2 and endocrine therapy instead of chemotherapy.

- Lapatinib and capecitabine should be considered for those patients who have progressed while on adjuvant trastuzumab and have evidence of brain metastases or for those do not have a significant response or have a shortened progression-free survival with chemotherapy and trastuzumab.

- Dramatic developments have occurred in the management of HER2-positive metastatic breast cancer in the past two decades. New regimens must focus not only on improved efficacy but also on superior toxicity profiles compared with current standard options. 
SUMMARY The use of targeted therapy directed against HER2 is currently the standard of care in patients with metastatic HER2-positive breast cancer. The combination of trastuzumab with a taxane as first-line treatment in HER2-positive metastatic breast cancer patients is the most common therapeutic approach in this population. The combination of trastuzumab with other chemotherapeutic agents, including vinorelbine and capecitabine; and hormonal therapy agents, such as aromatase inhibitors, have also demonstrated significant activity, and may be considered as an option for selected patients. Recently, the addition of pertuzumab to trastuzumab and docetaxel in first-line therapy has demonstrated an increased progression-free survival in HER2-positive metastatic breast cancer patients. Novel strategies against HER2 in first-line treatment or after progression include HER tyrosine kinase inhibitors such as lapatinib in combination with either chemotherapy, aromatase inhibitors or trastuzumab. An increasing list of new compounds are currently under investigation, such as trastuzumab-emtansine, afatinib, everolimus and antiangiogenic agents, among others. This review discusses potential therapeutic approaches in the first-line setting and after progression beyond trastuzumab in metastatic breast cancer HER2-positive tumors based on the latest evidence.

HER2 (ErbB2/neu) is a member of a family of transmembrane tyrosine kinase receptors that includes HER1 (the EGF receptor [EGFR]), HER3 (ErbB3) and HER4 (ErbB4). HER2 overexpression induces proliferation by disrupting the function of proteins that regulate cell cycle progression and apoptosis [1].

In the era before HER2-targeted therapy, the HER2-enriched subtype carried a poor prognosis; however, since the commercial availability of trastuzumab in 1998 and its routine incorporation in the management of metastatic breast cancer (MBC) and as adjuvant therapy in 2005, along with the development and integration of other HER2-targeted therapies, the history and evolution of this breast cancer subtype have changed dramatically [2]. Despite the success of targeted therapies in the treatment of metastatic HER2-positive breast cancer, many patients do not respond to trastuzumab therapy or progress after initiating trastuzumab and, eventually, the majority of patients will progress [3].

This review discusses potential therapeutic approaches that, according to recent data, may improve clinical outcomes in HER2-positive MBC, with special emphasis on those patients who have progressed on trastuzumab treatment.

Role of trastuzumab to date

Trastuzumab (Herceptin ${ }^{\circledR}$ ) is a recombinant humanized monoclonal IgG1 antibody that selectively binds to the receptor HER2 to inhibit the growth of tumor cells [4]. Trastuzumab has been shown in preclinical models to have synergistic activity with a variety of chemotherapeutic drugs. The mechanism of trastuzumab action is the subject of debate and several possibilities have been hypothesized [5].

HER2 overexpression leads to activation of the PI3K and the serine/threonine kinase Akt (also known as PKB) signal cascades, turnover of cyclin D1 and, as a result, cell cycle progression [6].

Downstream effects of the PI3K-Akt pathway also include inhibition of transcription of $p 27$ (a $\mathrm{Cdk} 2$ inhibitor). Trastuzumab increases nuclear and cytosolic levels of p27, thereby leading to cell cycle arrest (cytostatic effect) [5,7].

Trastuzumab has not only cytostatic but also cytotoxic properties. At least in part, these two properties may be due to the activation of antibody-dependent cellular cytotoxicity. There are many other possible mechanisms of trastuzumab action described; however, despite years of preclinical and clinical investigation the precise trastuzumab mechanism of action is not fully understood [8].

In the first-line HER2-positive MBC setting, Phase II studies of trastuzumab monotherapy have demonstrated an objective response rate (ORR) of $26 \%$ (95\% CI: 18.2-34.4\%), a clinical benefit rate (CBR) of $50 \%$ and a median duration of survival of 22.9 months [9]. In the pivotal Phase III trial conducted by Slamon et al., the addition of trastuzumab to chemotherapy resulted in a significantly improved time to progression (TTP) (median: 7.4 vs 4.6 months; $\mathrm{p}<0.001$ ), a higher ORR ( 50 vs 32\%; $<<0.001$ ), a longer duration of response (median: 9.1 vs 
6.1 months; $\mathrm{p}<0.001)$ and improved survival (median: 25.1 vs 20.3 months; $\mathrm{p}=0.01$ ). Among the patients who received trastuzumab and paclitaxel, the overall response was 38\% in comparison with $16 \%$ in patients treated with paclitaxel alone [10].

The results obtained with paclitaxel and trastuzumab were confirmed in a randomized Phase II study comparing docetaxel versus docetaxel with trastuzumab. This study demonstrated an improvement in the ORR in the trastuzumab arm (61 vs 34\%; $\mathrm{p}=0.0002$ ), progression-free survival (PFS; 11.7 vs 6.1 months; $\mathrm{p}=0.0001)$ and overall survival (OS; 31.2 vs 22.7 months; $\mathrm{p}=0.0325$ ) [11]. This pivotal data helped establish the use of taxanes and trastuzumab as the standard of care in the first-line treatment of HER2-positive MBC patients [11,12].

Platinum-based combinations with trastuzumab have also been evaluated in clinical trials and are associated with an improved ORR and a significant improvement in PFS, with no improvement in OS but an increased grade III-IV hematologic toxicity [13-17].

Vinorelbine in combination with trastuzumab has recently been shown to have a significant benefit and is now a standard first-line chemotherapy option for MBC patients. This combination was explored in the HERNATA trial, a Phase III study comparing trastuzumab plus vinorelbine versus trastuzumab plus docetaxel. This study showed no statistical difference in the response rate (RR), PFS or OS between both arms. However, the vinorelbine plus trastuzumab arm demonstrated a more favorable toxicity profile [18].

This lack of difference in efficacy between taxane and vinorelbine, both in combination with trastuzumab, was found in the TRAVIOTA study. In this study, the RR was 51 and $40 \%$ for the vinorelbine plus trastuzumab arm and the taxane plus trastuzumab arm, respectively (Fisher's exact test; $\mathrm{p}=0.37$ ). The median TTP was 8.5 and 6.0 months for the vinorelbine- and taxane-based arms, respectively (log-rank test; $\mathrm{p}=0.09$ ) [19]. The HERNATA and TRAVIOTA studies have helped establish the combination of vinorelbine plus trastuzumab as an effective first-line treatment option with a favorable toxicity profile. A list of Phase III clinical trials using HER2-targeted therapy in the first-line HER2-positive MBC setting is summarized in Table 1.
Anthracyclines alone are also particularly active in this patient cohort. In the anthracycline plus trastuzumab arm of the pivotal trial conducted by Slamon et al. in HER2-positive MBC, patients were treated for a planned duration of six cycles (cumulative anthracycline dose $360 \mathrm{mg} / \mathrm{m}^{2}$ ), with further cycles administered at investigator discretion. With this, the incidence of cardiac dysfunction and New York Heart Association class III-IV cardiotoxicity was 27 and 16\%, respectively. While the toxicity was too prohibitive, the best outcomes were obtained in this combination arm. After the risk of cardiotoxicity was recognized the concurrent administration with anthracyclines was avoided in clinical practice $[10,20]$. In recent trials of trastuzumab combined with chemotherapy or hormonal therapy, the incidence of cardiac events is in the range of $1-3 \%$ [21]. In the HERCULES trial, a prospective Phase I/II study of HER2-positive MBC, patients who received first-line trastuzumab plus cyclophosphamide and epirubicin showed acceptable dose-limiting cardiotoxicity [22]. These findings must be confirmed in further studies. There is a need for more data on the use of combinations of anthracyclines with trastuzumab in the metastatic setting and, in the meantime, this approach should only be utilized in a clinical trial setting.

\section{Trastuzumab beyond disease \\ progression}

The majority of patients with $\mathrm{MBC}$, who initially respond to trastuzumab, develop resistance within 1 year of treatment initiation, and in the adjuvant setting $15 \%$ of patients still relapse despite trastuzumab-based therapy [23].

Several mechanisms of trastuzumab resistance have been proposed, such as loss of PTEN function [8]. This is seen in 20-25\% of HER2-positive breast cancers and, according to Nagata et al., patients with PTEN-deficient tumors had significantly poorer RRs to trastuzumab-based therapy than those with normal PTEN. Thus, PTEN deficiency could be a predictor for trastuzumab resistance [24]. Data suggest that the accumulation of truncated forms of the HER2 receptor that lack the extracellular trastuzumab-binding domain known as p95 may also lead to resistance to trastuzumab, as trastuzumab is unable to bind to the cancer cell [24]. Recent literature suggests that very high levels of total HER2 protein expression may lead to de novo resistance to trastuzumab [25]. Another mechanism suggested 
Review | Landaverde \& Verma

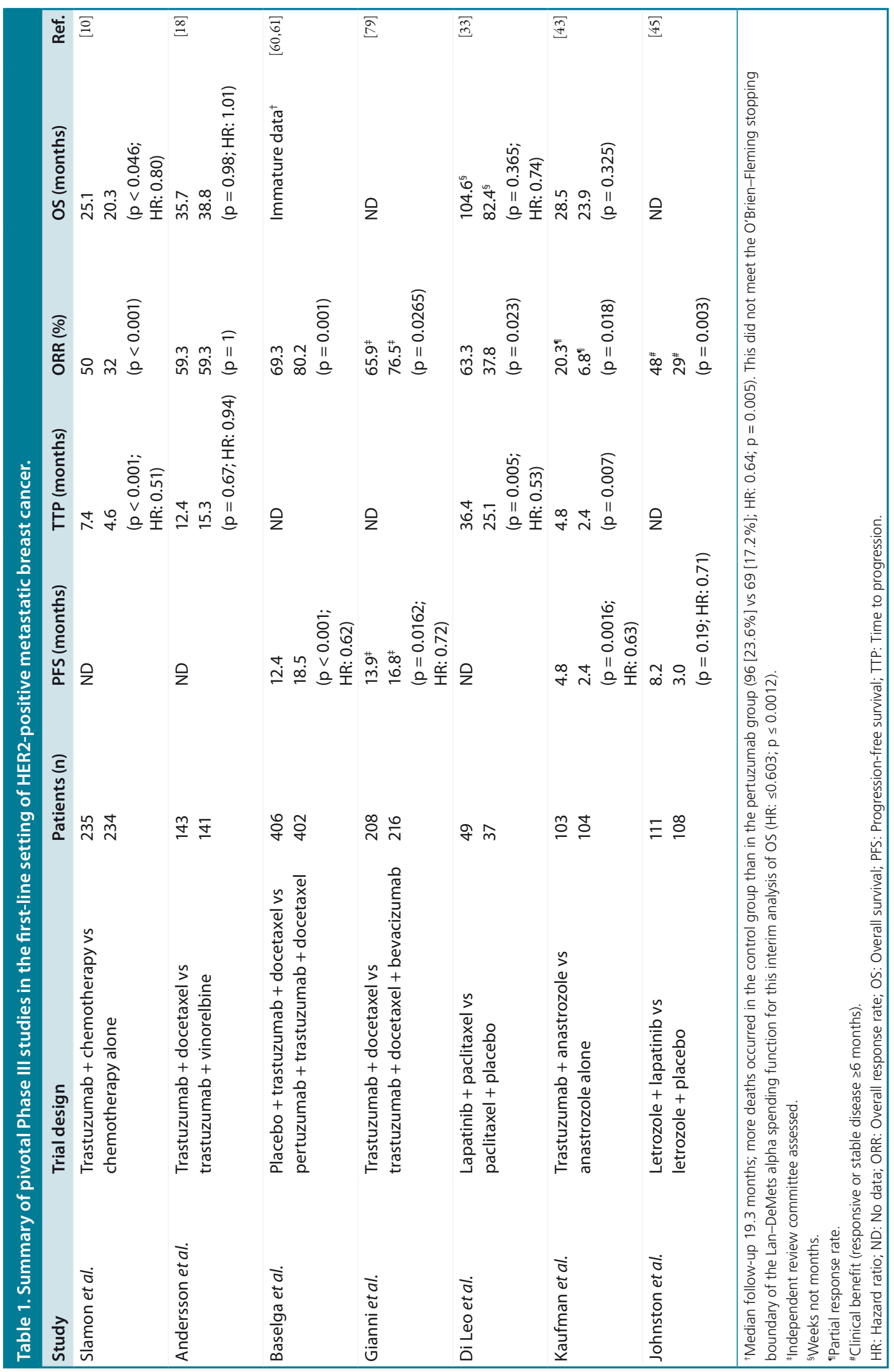


for resistance to trastuzumab is the increased signaling from the IGF-I receptor [26].

Recent advances in molecular biology are improving the understanding of the mechanism of primary or secondary resistance to trastuzumab. While these proposed mechanisms are of great scientific interest, there is no predictive biomarker that is available for clinical use to help predict intrinsic resistance to trastuzumab.

Over the past decade there has been emerging evidence on the benefit of trastuzumab in patients who have previously progressed on trastuzumab-based therapy. Initially, Phase II trials suggested continued benefit of trastuzumab beyond progression. This was validated in a Phase III trial (GBG 26) conducted by von Minckwitz et al., in which HER2-positive MBC patients who had progressed on a trastuzumab and chemotherapy combination were randomly assigned to receive capecitabine alone or capectabine plus continued trastuzumab. The combination arm was associated with a significantly longer median TTP (8.2 vs 5.6 months) and a nonstatistically significant improvement in OS (25.5 vs 20.4 months) [27]. This study showed that there are some patients that derive benefit from the continuation of trastuzumab beyond progression. Of note, this trial was stopped early due to poor accrual and, as such, may not be adequately powered to truly answer the question on the magnitude of benefit seen with trastuzumab beyond progression [28].

In the Blackwell et al. Phase III trial, 296 patients with HER2-positive MBC who progressed on one or more prior trastuzumabbased regimens were randomized to receive trastuzumab plus lapatinib (a dual HER1/ HER2 tyrosine kinase inhibitor [TKI], which is discussed later in this review) or lapatinib alone. This study demonstrated a significant improvement in median PFS (12 vs 8 weeks; hazard ratio [HR]: 0.73; 95\% CI: 0.57-0.93) and CBR (24.7 vs $12 \%)$. There was also a strikingly significant improvement in OS (9.5 vs 14.0 months) [29]. In conjunction, these two randomized Phase III trials support the benefit of continued trastuzumab for patients who have previously progressed on trastuzumab-based combination(s).

Lapatinib

Lapatinib is a reversible, dual TKI of the receptors HER1 and HER2. Inactivation of HER1/2 leads to the inhibition of downstream signaling, including PI3K-Akt and MAPK pathways. In multiple breast cancer cell lines, lapatinib can produce cell cycle arrest and a subsequent induction of apoptosis [30].

This inhibition was most prominent in tumors with activated ErbB receptors, including HER1 and HER2 [31]. In cells overexpressing HER2, lapatinib produced inhibition of cell proliferation inducing cell cycle arrest and apoptosis [32].

This compound has been evaluated in firstline treatment of HER2-positive MBC in a Phase III trial, which was largely conducted in a HER2-untested or -negative patient population with an inadvertent subset of only 86 HER2positive patients (15\%). The subgroup analysis of the HER2-positive subset revealed that the treatment with paclitaxel plus lapatinib resulted in statistically significant improvements in the TTP (36.4 vs 25.1 weeks), event-free survival (35.1 vs 21.9 weeks), ORR (63.3 vs $37.8 \%$ ) and CBR (69.4 vs $40.5 \%$ ) compared with paclitaxel plus placebo. This study failed to show an improvement in OS [33]. At the time of writing this article, lapatinib had not yet been approved by the US FDA in combination with paclitaxel as first-line treatment in HER2-positive MBC.

Lapatinib has mainly been explored after progression to chemotherapy and trastuzumab. In the pivotal Phase III trial, patients who had progressed on trastuzumab in a MBC setting were randomized to receive lapatinib plus capecitabine versus capecitabine alone. The median TTP, reported initially in the New England Journal of Medicine in 2006, was 8.4 months in the combination therapy group compared with 4.4 months in the monotherapy group [34]. At a planned interim analysis reported in 2008, the TTP was a median of 6.2 versus 4.3 months, which was significant in favor of the combination arm [35]. The mature and final analysis was reported in 2010 and the median OS times were 75.0 weeks for the combination arm and 64.7 weeks for the monotherapy arm (HR: 0.87; 95\% CI: $0.71-1.08 ; \mathrm{p}=0.210$ ), which was not statistically significant. However, the exclusion of crossover patients from the analysis resulted in a significant improvement in the median OS, with OS times of 75.0 weeks in the combination group and 56.4 weeks in the monotherapy group (HR: 0.78; 95\% CI: 0.62-0.97; $\mathrm{p}=0.023)$. Furthermore, the patients in the combination arm had fewer brain metastases in an unplanned exploratory analysis [36]. 
The other Phase III trial supporting the use of lapatinib beyond progression is with combination of the two HER2-targeted agents, lapatinib and trastuzumab, as discussed above (Blackwell et al. [29]). These two Phase III trials using lapatinib, along with the GBG 26 trial [27] evaluating trastuzumab in combination with capecitabine, demonstrate strong evidence that patients with HER2-positive disease derive clear benefit with continuous anti-HER2 therapy even upon progression.

\section{- Lapatinib \& brain metastasis}

Lapatinib is a small and lipophilic molecule that owing to these properties may cross the bloodbrain barrier. Lapatinib has also shown activity in brain metastases (BM) in preclinical studies. Using ${ }^{14} \mathrm{C}$-lapatinib in immunocompromised mice, studies have demonstrated an elevated concentration of this compound in BM [37]. This finding has also been recognized in two Phase II clinical trials investigating the benefit of lapatinib as monotherapy in HER2-positive MBC with BM in patients who progressed after cranial radiation and trastuzumab. Lapatinib was associated with a volumetric reduction in tumor size in these studies $[38,39]$. There is an increasing amount of data suggesting that the combination of lapatinib plus capecitabine is very active in HER2-positive MBC with $\mathrm{BM}$; this therapeutic approach may improve OS when compared with trastuzumab-based therapies in this particular setting $[40,41]$.

Treatment of hormone receptor-positive \& HER2-positive MBC: evidence for combined aromatase inhibitor with anti-HER2 treatment

Several models in breast cancer cells suggest that estrogen receptors can be activated by HER family members in a bidirectional crosstalk. This phenomenon can generate the activation of many intracellular pathways including metalloproteinases, tyrosine kinase cascades, MAPK and PI3KAKT pathways among others. These downstream activated kinases will phosphorylate and activate estrogen receptors augmenting the activities of estrogen receptor and HER family members as well as other kinase-related pathways [42]. This crosstalk plays a role not only in the endocrine response but also in endocrine therapy resistance. This section discusses the clinical evidence for combined hormonal therapy with anti-HER2 treatment.
The TAnDEM study compared trastuzumab plus anastrozole versus anastrozole alone, and showed an improvement in PFS in the trastuzumab plus anastrozole arm (2.4 vs 4.8 months; HR: 0.63 ; 95\% CI: $0.47-0.84 ; \mathrm{p}=0.016)$. In patients with centrally confirmed hormone receptor positivity $(\mathrm{n}=150)$, the median PFS was 5.6 and 3.8 months in the trastuzumab plus anastrozole and anastrozole alone arms, respectively $(\log$-rank; $\mathrm{p}=0.006)$. No difference in OS was seen between the two arms; however, $70 \%$ of patients in the anastrozole alone arm received trastuzumab upon progression [43]. Another trial investigated the combination of trastuzumab and letrozole and found similar results [44].

Lapatinib has been studied in combination with letrozole as first-line treatment in HER2-/ hormone receptor-positive $\mathrm{MBC}$ tumors and this approach was evaluated in the EGF30008 trial. In this Phase III trial, the addition of lapatinib to letrozole significantly reduced the risk of disease progression versus letrozole plus placebo (HR: 0.71; 95\% CI: 0.53-0.96; $\mathrm{p}=0.019)$ and the median PFS was 8.2 v 3.0 months, respectively. The CBR was significantly greater for lapatinib plus letrozole versus letrozole plus placebo ( 48 vs $29 \%$, respectively; odds ratio: $0.4 ; 95 \% \mathrm{CI}$ : $0.2-0.8 ; \mathrm{p}=0.003)$. The ORR was also significantly higher in lapatinib-treated patients (28 vs $15 \%$; odds ratio: 0.4 ; $95 \%$ CI: $0.2-0.9$; $\mathrm{p}=0.021)[45,46]$.

To date there is no head-to-head comparison trial of chemotherapy versus endocrine therapy along with anti-HER 2 treatment to suggest the most optimal regimen. On review of the evidence there is a greater benefit seen with chemotherapy and an anti-HER 2 approach; however, this comes with increased toxicity. The option of antiestrogen treatment along with anti-HER2 therapy could be considered in patients with low disease volume, such as patients with only bone metastases and those with a long disease-free interval, suggesting a slower rate of disease progression. This approach is based mainly on clinical judgment since there are no prospective data and no evidence for biomarkers to determine which patients will derive real benefit from anti-HER2 in combination with endocrine therapy [47-52].

\section{Targeting ErbB family receptors:}

new drugs

Amplification and/or overexpression of the three other HER-related family members may 
also influence the clinical course and outcomes of HER2-positive breast cancer. Overexpression of HER1 and HER3 significantly reduces disease-specific survival [53].

It is possible that targeting those receptors at the same time or by a different mechanism could translate into clinical benefit by potentially overcoming mechanisms of resistance and blocking the significant crosstalk among the kinase pathways.

There are data suggesting that targeting HER2 can increase the expression of HER 3 and increase the possibility of HER2-HER3 heterodimerization, leading to resistance to trastuzumab and subsequently cancer progression [54,55]. In addition to functioning as a homodimer, HER2 is the preferred partner for the other EGFR family proteins, particularly HER3, and HER2 heterodimers appear to be more active than HER2 homodimers; this event can produce increased tumor proliferation [56].

\section{- Pertuzumab}

Pertuzumab is a humanized monoclonal antibody that binds to HER2 near the center of domain II, a domain required for interactions with other EGFR family members, especially HER3, thereby preventing dimerization of HER2 with other HER receptors (HER3, HER1 and HER4) [57,58]. This drug has been studied in a multicenter, open-label, single-arm Phase II study. Sixty six patients with advanced HER2-positive breast cancer in whom disease progression had occurred during prior trastuzumab-based therapy received trastuzumab plus pertuzumab. The ORR was $24.2 \%$ and the CBR was $50 \%$. The median PFS was 5.5 months. Overall, the combination of pertuzumab plus trastuzumab was well tolerated, and adverse events were mild to moderate. This study showed that the combination of pertuzumab plus trastuzumab is active in patients with metastatic HER2-positive breast cancer who had experienced progression during prior trastuzumab therapy [59].

Recently, results from a pivotal trial evaluating pertuzumab, CLEOPATRA, were published. This is an international, randomized, doubleblind, placebo-controlled Phase III trial, in which patients were randomized (1:1) to receive either docetaxel, trastuzumab and pertuzumab or docetaxel, trastuzumab and placebo. The primary end point of the study was PFS. Results demonstrate that the pertuzumab-containing arm had an improved PFS (18.5 vs 12.4 months in a control group), an absolute improvement of more than 6 months $(\mathrm{p}<0.0001)$ compared with the control arm. In terms of OS, with a median follow-up of 19.3 months (immature data), more deaths occurred in the control group than in the pertuzumab group (96 [23.6\%] vs 69 [17.2\%] deaths; HR: 0.64; $\mathrm{p}=0.005)[60,61]$. This is clearly a significant benefit in PFS and probably in OS, establishing a new standard of care in the first-line setting for selected patients. However, this is a very expensive combination and more information is needed on which patients are more likely to derive benefit from such an approach.

\section{- Trastuzumab-emtansine}

Trastuzumab-emtansine (T-DM1) is an antibody-drug conjugate combining trastuzumab with a chemotherapeutic antimicrotubule agent derivative of maytansanine (DM1). The anatomy of this antibody-cytotoxic conjugate is divided in three components: the antibody itself, the cytotoxic agent and, importantly, the linker molecule [62-64]. Activation of cytotoxicity of this conjugate requires internalization into the cell after binding to HER2 [65].

In the TDM4258g Phase II study, in patients with HER2-positive MBC who had tumor progression after prior treatment with HER2directed therapy and who had received prior chemotherapy, with a follow-up of 12 months among 112 treated patients, T-DM1 demonstrated an ORR of $25.9 \%$ (95\% CI: 18.4-34.4\%). The median PFS was 4.6 months (95\% CI: 3.9-8.6 months). T-DM1 was well tolerated with no dose-limiting cardiotoxicity [66].

At the 2011 European Multidisciplinary Cancer Congress, the primary efficacy and updated safety results of the open-label Phase II study (TDM4450g/BO21976) of T-DM1 versus trastuzumab plus docetaxel in previously untreated HER2-positive MBC were presented. With respect to efficacy data, this study showed a significant improvement in PFS in the T-DM1 $\operatorname{arm}(14.2$ vs 9.2 months; HR: 0.59; $\mathrm{p}=0.035)$. Overall, T-DM1 was very well tolerated and had a much more favorable toxicity profile compared with docetaxel and trastuzumab [67].

This compound is now being studied in three key trials. EMILIA (NCT00829166) is an openlabel Phase III trial evaluating T-DM1 versus 
capecitabine plus lapatinib in trastuzumabpretreated MBC patients $[68,101]$. Another study, recently closed to patient accrual, MARIANNE (NCT01120184), is a Phase III trial evaluating T-DM1, T-DM1 plus pertuzumab or trastuzumab plus a taxane as first-line therapy for HER2-positive MBC [58,102]. The third Phase III trial with T-DM1, THERESA (NCT01419197), now open, will compare single-agent T-DM1 versus physicians' therapy of choice for patients in the third-line (or greater) setting for HER2positive $\mathrm{MBC}$ who have been previously treated with anthracyclines, capecitabine, taxanes, trastuzumab and lapatinib $[69,103]$.

\section{- Neratinib}

Neratinib is an oral, irreversible, inhibitor of HER1, HER2 and HER4 [70]. Burstein et al. conducted a Phase II trial with neratinib in HER2positive MBC. They found that the 16-week PFS rates were $59 \%$ for patients with prior trastuzumab treatment and $78 \%$ for patients with no prior trastuzumab therapy. The median PFS was 22.3 and 39.6 weeks, respectively. The ORR was $24 \%$ among patients with prior trastuzumab treatment and $56 \%$ in the trastuzumab-naive cohort. Nausea, diarrhea, vomiting and fatigue were the most common adverse events reported [71]. A Phase II clinical trial was presented at the 2011 San Antonio Breast Cancer Symposium by Martin et al.; neratinib was found to be inferior to lapatinib plus capecitabine, with a median PFS of 4.5 versus 6.8 months $(p=0.23)$, and ORRs were substantially higher with lapatinib plus capecitabine versus neratinib (40 vs $29 \%$ ). The incidence of diarrhea was significantly higher with neratinib versus lapatinib plus capecitabine ( 85 vs $68 \% ; \mathrm{p}=0.002$ ) [72]. A Phase III trial is ongoing using neratinib in MBC: NCT00915018 is investigating paclitaxel with neratinib or combined with trastuzumab $[65,104]$.

\section{- Everolimus}

Everolimus (RAD001) is an inhibitor of mTOR, a serine/threonine protein kinase involved in the regulatory mechanisms of cancer cell proliferation. Everolimus is being extensively studied in HER2-positive MBC. In a Phase I study everolimus was combined with weekly paclitaxel plus trastuzumab in trastuzumab-resistant disease; the RR was $44 \%$, disease control for 6 months rate was $74 \%$ and median PFS was 34 weeks. This combination showed antitumor activity in patients with trastuzumab-pretreated and resistant metastatic disease [73].

Dalenc et al. also evaluated the activity of everolimus in a multicenter Phase II trial using paclitaxel plus trastuzumab with everolimus in patients whose disease was resistant to both trastuzumab and taxanes. The investigators observed five confirmed partial response (20\%), 14 stable disease $(56 \%)$ and six progressive disease $(24 \%)$. Treatment was well tolerated. In conclusion, this combination showed important activity in patients resistant to lapatinib therapy [74]. Recently published data from a Phase I/IIb trial by Morrow et al. evaluated the combination of everolimus and trastuzumab in patients with HER2-overexpressing MBC who progressed on trastuzumab-based therapy. The CBR was 34\%, the median PFS was 4.1 months and they concluded that the inhibition of mTOR results in clinical benefit and disease response in patients with trastuzumab-resistant HER2-overexpressing MBC [75].

The clinical adoption of everolimus in HER2positive $\mathrm{MBC}$ patients will depend on the results from two ongoing pivotal Phase III clinical trials. The first study is BOLERO-1 (NCT00876395; currently closed to accrual), a Phase III, randomized, double-blind study of everolimus plus trastuzumab and paclitaxel as upfront therapy in women with HER2-positive locally advanced breast cancer or MBC [105]. Patients are randomized to receive either everolimus or placebo, plus trastuzumab and paclitaxel. The primary end point is PFS, and the secondary main end points are OS, ORR and CBR. The second study is BOLERO-3 (NCT01007942), a Phase III, randomized, double-blind study of everolimus plus trastuzumab and vinorelbine in women with HER2-positive locally advanced breast cancer or MBC previously treated with a taxane and resistant to trastuzumab $[62,106]$.

\section{- Afatinib}

Afatinib is an irreversible inhibitor of HER1 and HER2. Afatinib activity in HER2-positive MBC has been evaluated in an open-label Phase II study in which patients received afatinib as monotherapy after failure of treatment to trastuzumab. CBR (complete response plus partial response plus stable disease) was observed in $53 \%$ of patients $(n=41)[76]$. The LUX-Breast 1 study (NCT01125566) is evaluating the use of afatinib or trastuzumab with vinorelbine in 
HER2-positive MBC patients who progressed after trastuzumab [107].

\section{- Antiangiogenic therapy}

Many molecules targeting the VEGF or VEGF receptor are under investigation in HER2positive MBC, since overexpression of HER 2 has been shown to be associated with the increased angiogenesis and expression of VEGF in tumor cells. For example, sunitinib in a Phase II trial in patients with $\mathrm{MBC}$ previously treated with an anthracycline and a taxane was found to produce notable responses in HER2-positive, trastuzumab-treated patients [77,78].

Bevacizumab was studied in the Phase III trial AVEREL (NCT00391092), a randomized, open-label study designed to compare first-line treatment with bevacizumab plus trastuzumab plus docetaxel versus trastuzumab plus docetaxel alone with a primary end point of investigatorassessed PFS in patients with HER2-positive locally recurrent or MBC [108]. The AVEREL

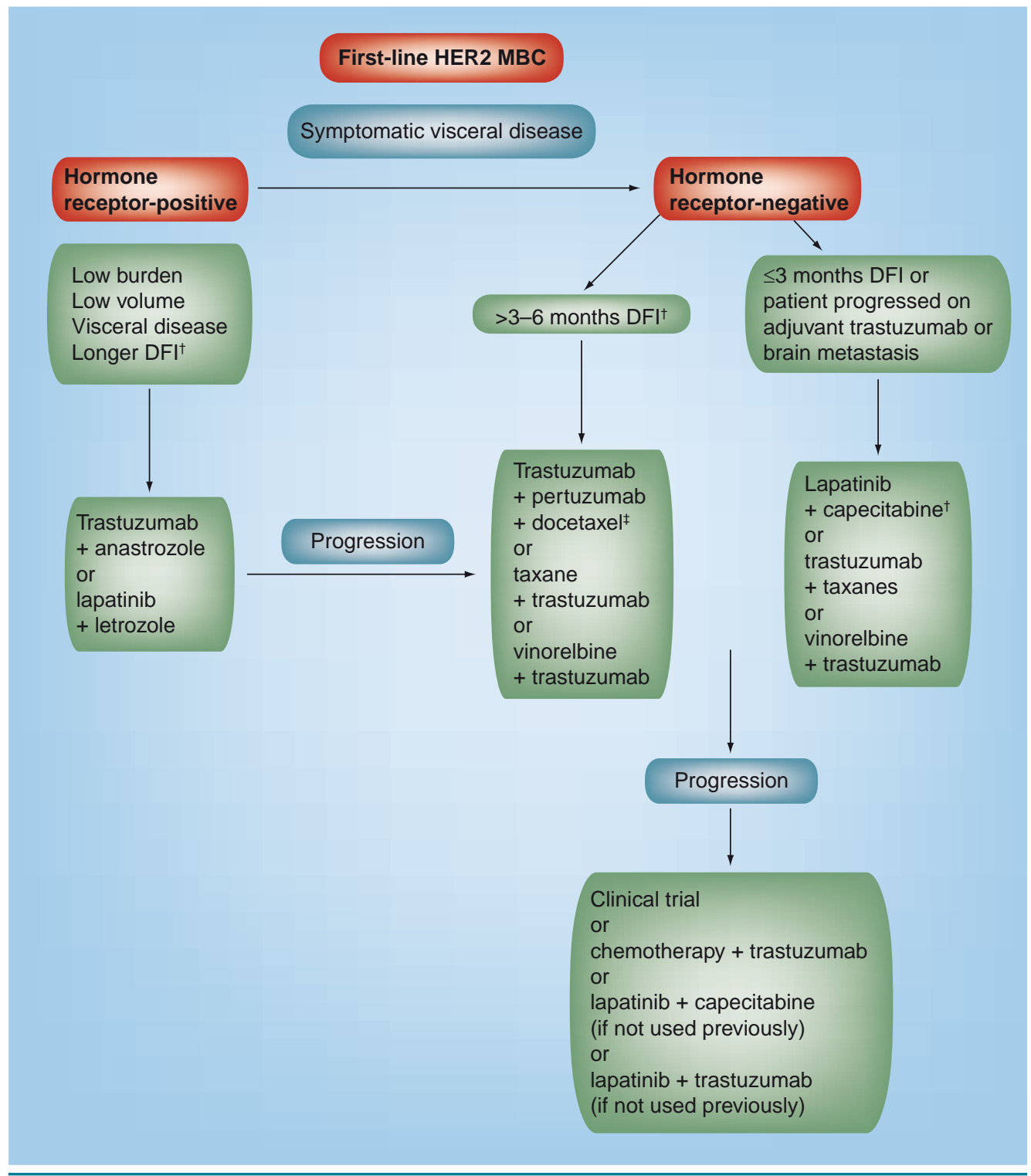

Figure 1. Treatment algorithm for HER2-positive metastatic breast cancer.

This approach based on disease-free interval is primarily based on clinical judgment.

*This regimen is not currently approved by the US FDA.

DFI: Disease-free interval; MBC: Metastatic breast cancer. 
study was presented recently at the 2011 San Antonio Breast Cancer Symposium; it demonstrated a nonstatistically significant improved PFS when bevacizumab was combined with trastuzumab plus docetaxel. The investigatorassessed PFS had a HR of 0.82 ( $\mathrm{p}=0.0775)$ and the independent review committee-assessed PFS had a HR of 0.72 ( $p=0.0162)$ [79]. Given the marginal benefit in the overall population, efforts are now underway to determine a specific biomarker to identify the subgroups most likely to benefit.

\section{- Heat shock protein inhibitors}

In preclinical models the Hsp90 chaperone complex is important for the stability, maturation and activation of important oncoproteins such as HER2, HER1, Akt and MAPK, among others. These models have demonstrated that Hsp90 inhibition produces proteosomal degradation of HER2, which is enhanced with the addition of trastuzumab. Currently these inhibitors are under clinical evaluation [80].

\section{Future perspective}

There are an increasing number of effective therapeutic options available for HER2-positive MBC. There are many therapies in development but, at this point, it is clear that we need continued suppression of HER2 activity either by a monoclonal antibody, TKI or combination of the two. A clinical uncertainty still remains as to which is the more superior HER2-targeted therapy and the need for chemotherapy with combined HER2-targeted therapy.

There is a clear interest in utilizing a combined targeted approach (either with a TKI plus trastuzumab or trastuzumab plus pertuzumab); however, this is quite costly and we need more information on which patients are more likely to respond to such an approach.

In addition, further biomarker research is needed to better understand mechanisms of resistance to help us personalize a treatment approach upon disease progression.

The authors propose an algorithm of treatment based mainly on Phase III trials according to the data available, which also integrates an approach from our clinical practice (Figure 1). The clinician in the context of multidisciplinary team needs to choose the right drug/combination based on understanding of disease biology and appropriate risk-benefit analysis.

Financial \& competing interests disclosure $S$ Verma is a researcher for Sanofi-Aventis and Roche. $S$ Verma is also on advisory boards for GSK, Roche and Sanofi-Aventis. The authors have no other relevant affiliations or financial involvement with any organization or entity with a financial interest in or financial conflict with the subject matter or materials discussed in the manuscript apart from those disclosed.

No writing assistance was utilized in the production of this manuscript.

\section{References}

Papers of special note have been highlighted as:

- of interest

1. of considerable interest

1 Park JW, Neve RM, Szollosi J, Benz CC. Unraveling the biologic and clinical complexities of HER2. Clin. Breast Cancer 8, 392-401 (2008).

2 Carey LA. Through a glass darkly: advances in understanding breast cancer biology, 2000-2010. Clin. Breast Cancer 10(3), 188-195 (2010).

3 Jackish C. Challenges in the treatment of ErbB2 (HER2)-positive breast cancer. Eur. J. Cancer Suppl. 6(5), 7-14 (2008).

4 Park IH, Ro J, Lee KS, Nam BH, Kwon Y, Shin KH. Trastuzumab treatment beyond brain progression in HER2-positive metastatic breast cancer. Ann. Oncol. 20 (1), 56-62 (2009).
5 Pal SK, Pegram M. HER2 targeted therapy in breast cancer...beyond herceptin. Rev. Endocr. Metab. Disord. 8(3), 269-277 (2007).

6 Delord JP, Allal C, Canal M et al. Selective inhibition of HER2 inhibits AKT signal transduction and prolongs disease-free survival in a micrometastasis model of ovarian carcinoma. Ann. Oncol. 16, 1889-1897 (2005).

7 Lane HA, Motoyama AB, Beuvink I, Hynes NE. Modulation of p27/Cdk2 complex formation through 4D5-mediated inhibition of HER2 receptor signaling. Ann. Oncol. 12(Suppl. 1), S21-S22 (2001)

8 Valabrega G, Montemurro F, Aglietta M. Trastuzumab: mechanism of action, resistance and future perspectives in HER2-overexpressing breast cancer. Ann . Oncol. 18(6), 977-984 (2007).
9 Vogel CL, Cobleigh MA, Tripathy D et al. Efficacy and safety of trastuzumab as a single agent in first-line treatment of HER2- overexpressing metastatic breast cancer. J. Clin. Oncol. 20, 719-726 (2002).

10 Slamon DJ, Leyland-Jones B, Shak S et al. Use of chemotherapy plus a monoclonal antibody against HER2 for metastatic breast cancer that overexpresses HER2. N. Engl. J. Med. 344(11), 783-792 (2001).

n This is a pivotal study in the management of HER2-positive metastatic breast cancer.

11 Marty M, Cognetti F, Maraninchi D et al. Randomized Phase II trial of the efficacy and safety of trastuzumab combined with docetaxel in patients with human epidermal growth factor receptor 2-positive metastatic breast cancer administered as first-line treatment: the M77001 study group. J. Clin. Oncol. 23(19), 4265-4274 (2005). 
12 Seidman AD, Berry D, Cirrincione C et al. Randomized Phase III trial of weekly compared with every-3-weeks paclitaxel for metastatic breast cancer, with trastuzumab for all HER-2 overexpressors and random assignment to trastuzumab or not in HER-2 non overexpressors: final results of Cancer and Leukemia Group B protocol 9840. J. Clin. Oncol. 26(10), 1642-1649 (2008).

13 Valero V, Forbes J, Pegram MD et al. Multicenter Phase III randomized trial comparing docetaxel and trastuzumab with docetaxel, carboplatin, and trastuzumab as first-line chemotherapy for patients with HER2-gene-amplified metastatic breast cancer (BCIRG 007 study): two highly active therapeutic regimens. J. Clin. Oncol. 29(2), 149-156 (2011).

14 Pegram MD, Pienkowski T, Northfelt DW et al. Results of two open-label, multicenter Phase II studies of docetaxel, platinum salts, and trastuzumab in HER2-positive advanced breast cancer. J. Natl Cancer Inst. 96(10), 759-769 (2004).

15 Jenkins RB, Visscher DW, Thrower AR et al. Two concurrent Phase II trials of paclitaxel/carboplatin/trastuzumab (weekly or every-3-week schedule) as first-line therapy in women with HER2-overexpressing metastatic breast cancer: NCCTG study 983252. Clin. Breast Cancer 6(5), 425-432 (2005).

16 Robert N, Leyland-Jones B, Asmar L et al. Randomized Phase III study of trastuzumab, paclitaxel, and carboplatin compared with trastuzumab and paclitaxel in women with HER-2-overexpressing metastatic breast cancer. J. Clin. Oncol. 24(18), 2786-2792 (2006).

17 Stemmler HJ, Kahlert S, Brudler O et al. High efficacy of gemcitabine and cisplatin plus trastuzumab in patients with HER2-overexpressing metastatic breast cancer: a Phase II study. Clin. Oncol. (R. Coll. Radiol.) 17(8), 630-635 (2005).

18 Andersson M, Lidbrink E, Bjerre K et al. Phase III randomized study comparing docetaxel plus trastuzumab with vinorelbine plus trastuzumab as first-line therapy of metastatic or locally advanced human epidermal growth factor receptor 2-positive breast cancer: the HERNATA study. J. Clin. Oncol. 29(3), 264-271 (2011).

- This trial generated a new treatment option for first-line treatment of HER2-positive metastatic breast cancer.

19 Burstein HJ, Keshaviah A, Baron AD et al. Trastuzumab plus vinorelbine or taxane chemotherapy for HER2-overexpressing metastatic breast cancer: the trastuzumab and vinorelbine or taxane study. Cancer 110 , 965-972 (2007).

20 Rayson D, Richel D, Chia S, Jackisch C, van der Vegt $S$, Suter T. Anthracyclinetrastuzumab regimens for HER $2 /$ neuoverexpressing breast cancer: current experience and future strategies. Ann. Oncol. 19(9), 1530-1539 (2008).

21 Cook-Bruns N. Retrospective analysis of the safety of herceptin immunotherapy in metastatic breast cancer. Oncology 61(Suppl. 2), 58-66 (2001).

22 Untch M, Muscholl M, Tjulandin S et al. First-line trastuzumab plus epirubicin and cyclophosphamide therapy in patients with human epidermal growth factor receptor 2-positive metastatic breast cancer: cardiac safety and efficacy data from the Herceptin, Cyclophosphamide, and Epirubicin (HERCULES) trial. J. Clin. Oncol. 28(9), 1473-1480 (2010).

23 Nahta R, Yu D, Hung MC, Hortobagyi GN, Esteva FJ. Mechanisms of disease: understanding resistance to HER2-targeted therapy in human breast cancer. Nat. Clin. Pract. Oncol. 3(5), 269-280 (2006).

24 Nagata Y, Lan KH, Zhou X et al. PTEN activation contributes to tumor inhibition by trastuzumab, and loss of PTEN predicts trastuzumab resistance in patients. Cancer Cell 6(2), 117-127 (2004).

25 Bates M, Sperinde J, Kostler WJ et al. Identification of a subpopulation of metastatic breast cancer patients with very high HER2 expression levels and possible resistance to trastuzumab. Ann. Oncol. 22(9), 2014-2020 (2011).

26 Harbeck N, Pegram MD, Ruschoff J, Mobus V. Targeted therapy in metastatic breast cancer: the HER2/neu oncogene. Breast Care (Basel) 5(Suppl. 1), 3-7 (2010).

27 von Minckwitz G, du Bois A, Schmidt M et al. Trastuzumab beyond progression in human epidermal growth factor receptor 2-positive advanced breast cancer: a German Breast Group 26/Breast International Group 03-05 study. J. Clin. Oncol. 27(12), 1999-2006 (2009).

- Demonstrates the importance of HER2 suppression beyond progression.

28 Valabrega G, Aglietta M, Montemurro F. Trastuzumab beyond disease progression: case closed? J. Clin. Oncol. 27(27), e121-e122; author reply e124-e125 (2009).

29 Blackwell KL, Burstein HJ, Storniolo AM et al. Randomized study of lapatinib alone or in combination with trastuzumab in women with ErbB2-positive, trastuzumab-refractory metastatic breast cancer. J. Clin. Oncol. 28(7), 1124-1130 (2010).

- Showed that chemotherapy is probably not always needed and dual inhibition needs to be explored. It also showed that trastuzumab activity can be restored.

30 Rusnak DW, Lackey K, Affleck K et al. The effects of the novel, reversible epidermal growth factor receptor/ErbB-2 tyrosine kinase inhibitor, GW2016, on the growth of human normal and tumor-derived cell lines in vitro and in vivo. Mol. Cancer Ther. 1 , 85-94 (2001).

31 Xia W, Mullin RJ, Keith BR et al. Anti-tumor activity of GW572016: a dual tyrosine kinase inhibitor blocks EGF activation of EGFR/erbB2 and downstream Erk1/2 and AKT pathways. Oncogene 21, 6255-6263 (2002).

32 Amir E, Ocana A, Seruga B, Freedman O, Clemons M. Lapatinib and HER2 status: results of a meta-analysis of randomized Phase III trials in metastatic breast cancer. Cancer Treat. Rev. 36(5), 410-415 (2010).

33 Di Leo A, Gomez HL, Aziz Z et al. Phase III, double-blind, randomized study comparing lapatinib plus paclitaxel with placebo plus paclitaxel as first-line treatment for metastatic breast cancer. J. Clin. Oncol. 26(34), 5544-5552 (2008).

34 Geyer CE, Forster J, Lindquist D et al. Lapatinib plus capecitabine for HER2-positive advanced breast cancer. $N$. Engl. J. Med. 355(26), 2733-2743 (2006).

35 Cameron D, Casey M, Press M et al. A Phase III randomized comparison of lapatinib plus capecitabine versus capecitabine alone in women with advanced breast cancer that has progressed on trastuzumab: updated efficacy and biomarker analyses. Breast Cancer Res.Treat. 112(3), 533-543 (2008).

- This Phase III trial showed that the combination of lapatinib and capecitabine is effective after progression on trastuzumab-based regimens.

36 Cameron D, Casey M, Oliva C, Newstat B, Imwalle B, Geyer CE. Lapatinib plus capecitabine in women with HER-2-positive advanced breast cancer: final survival analysis of a Phase III randomized trial. Oncologist 15(9), 924-934 (2010).

37 Taskar KS, Rudraraju V, Mittapalli RK et al. Lapatinib distribution in HER2 overexpressing experimental brain metastases of breast cancer. Pharm. Res. 29, 770-781 (2012). 
38 Lin NU, Carey LA, Liu MC et al. Phase II trial of lapatinib for brain metastases in patients with human epidermal growth factor receptor 2-positive breast cancer. J. Clin. Oncol. 26, 1993-1999 (2008).

39 Lin NU, Dieras V, Paul D et al. Multicenter Phase II study of lapatinib in patients with brain metastases from HER2-positive breast cancer. Clin. Cancer Res. 15, 1452-1459 (2009).

40 Metro G, Foglietta J, Russillo M et al. Clinical outcome of patients with brain metastases from HER2-positive breast cancer treated with lapatinib and capecitabine. Ann. Oncol. 22, 625-630 (2011).

41 Bartsch R, Berghoff A, Pluschnig U et al. Impact of anti-HER2 therapy on overall survival in HER2-overexpressing breast cancer patients with brain metastases. Br. J. Cancer 106, 25-31 (2012).

42 Arpino G, Wiechmann L, Osborne KC, Schiff R. Crosstalk between the estrogen receptor and the HER tyrosine kinase receptor family: molecular mechanism and clinical implications for endocrine therapy resistance. Endocr. Rev. 29(2), 217-233 (2008).

43 Kaufman B, Mackey JR, Clemens MR et al. Trastuzumab plus anastrozole versus anastrozole alone for the treatment of postmenopausal women with human epidermal growth factor receptor 2-positive, hormone receptor-positive metastatic breast cancer: results from the randomized Phase III TAnDEM study. J. Clin. Oncol. 27(33), 5529-5537 (2009).

- Changed the paradigm of treatment for HER2-positive metastatic breast cancer, incorporating antiestrogen plus trastuzumab treatment instead of chemotherapy plus trastuzumab.

44 Marcom PK, Isaacs C, Harris L et al. The combination of letrozole and trastuzumab as first or second-line biological therapy produces durable responses in a subset of HER2 positive and ER positive advanced breast cancers. Breast Cancer Res. Treat. 102, 43-49 (2007).

45 Johnston S, Pippen J Jr, Pivot X et al. Lapatinib combined with letrozole versus letrozole and placebo as first-line therapy for postmenopausal hormone receptor-positive metastatic breast cancer. J. Clin. Oncol. 27(33), 5538-5546 (2009)

- Demonstrated that anti-HER2 compounds may be used in combination with aromatase inhibitors as first-line treatment.

46 Schwartzberg LS, Franco SX, Florance A, O’Rourke L, Maltzman J, Johnston S.
Lapatinib plus letrozole as first-line therapy for HER-2+ hormone receptor-positive metastatic breast cancer. Oncologist 15(2), 122-129 (2010).

47 Gluck S, Arteaga CL, Osborne CK Optimizing chemotherapy-free survival for the ER/HER2-positive metastatic breast cancer patient. Clin. Cancer Res. 17(17), 5559-5561 (2011).

48 Huober J, Fasching P, Paepke S et al. Letrozole in combination with trastuzumab is superior to letrozolemonotherapy as first line treatment in patients with hormone receptor positive, HER 2 positive metastatic breast cancer - results of the eLEcTRA trial. Cancer Res. 69(24 Suppl. 3), Abstract 4094 (2009).

49 Kemp Z, Jones A. A shift in the treatment of hormone receptor and human epidermal growth factor receptor 2-positive metastatic breast cancer. Adv. Ther. 28(8), 603-614 (2011)

50 Azim HA Jr, Piccart MJ. Simultaneous targeting of estrogen receptor and HER 2 in breast cancer. Expert Rev. Anticancer Ther. $10(8), 1255-1263$ (2010).

51 Harris CA, Ward RL, Dobbins TA, Drew AK, Pearson $S$. The efficacy of HER2-targeted agents in metastatic breast cancer: a meta-analysis. Ann. Oncol. 22(6), 1308-1317 (2011)

52 Cortes J, Saura C, Bellet M et al. HER2 and hormone receptor-positive breast cancer - blocking the right target. Nat. Rev. Clin. Oncol. 8(5), 307-311 (2011).

53 Wiseman SM, Makretsov N, Nielsen TO et al. Coexpression of the type 1 growth factor receptor family members HER-1, HER-2, and HER-3 has a synergistic negative prognostic effect on breast carcinoma survival. Cancer 103(9), 1770-1777 (2005).

54 Junttila TT, Akita RW, Parsons K et al. Ligand-independent HER2/HER3/PI3K complex is disrupted by trastuzumab and is effectively inhibited by the PI3K inhibitor GDC-0941. Cancer Cell 15(5), 429-440 (2009).

55 Lee-Hoeflich ST, Crocker L, Yao E et al. A central role for HER3 in HER amplified breast cancer: implications for targeted therapy. Cancer Res. 68(14), 5878-5887 (2008).

56 Ahn ER, CL Vogel. Dual HER2-targeted approaches in HER2-positive breast cancer. Breast Cancer Res. Treat. 131(2), 371-383 (2012)

57 Sheridan C. Pertuzumab to bolster Roche/Genentech's breast cancer franchise? Nat. Biotechnol. 29(10), 856-858 (2011).
58 Adams CW, Allison DE, Flagella K et al. Humanization of a recombinant monoclona antibody to produce a therapeutic HER dimerization inhibitor, pertuzumab. Cancer Immunol. Immunother. 55, 717-727 (2006).

59 Baselga, J, Gelmon KA, Verma S et al. Phase II trial of pertuzumab and trastuzumab in patients with human epidermal growth factor receptor 2-positive metastatic breast cancer that progressed during prior trastuzumab therapy. J. Clin. Oncol. 28(7), 1138-1144 (2010).

60 Baselga J, Kim SB, Im SA et al. A Phase III, randomized, double-blind, placebocontrolled registration trial to evaluate the efficacy and safety of pertuzumab + trastuzumab + docetaxel vs. placebo + trastuzumab + docetaxel in patients with previously untreated HER2-positive metastatic breast cancer (CLEOPATRA). Presented at: San Antonio Breast Cancer Symposium. San Antonio, TX, USA, 6-10 December 2011

61 Baselga J, Cortés J, Kim SB et al.; CLEOPATRA Study Group. Pertuzumab plus trastuzumab plus docetaxel for metastatic breast cancer. N. Engl. J. Med. 366, 109-119 (2012).

-n New paradigm to treat HER2 metastatic breast cancer; probably a practice-changing regimen.

62 Perez EA, Spano JP. Current and emerging targeted therapies for metastatic breast cancer. Cancer doi:10.1002/cncr.26356 (2011) (Epub ahead of print).

63 Lorusso PM, Weiss D, Guardino E, Girish S, Sliwkowski MX. Trastuzumab emtansine: a unique antibody-drug conjugate in development for human epidermal growth factor receptor 2-positive cancer. Clin Cancer Res. 17(20), 6437-6447 (2011).

64 Isakoff SJ, Baselga J. Trastuzumab-DM1: building a chemotherapy-free road in the treatment of human epidermal growth factor receptor 2-positive breast cancer. J. Clin. Oncol. 29, 351-354 (2011).

65 Saini KS, Azim Jr HA, Metzger-Filho O et al. Beyond trastuzumab: new treatment options for HER2-positive breast cancer. Breast 20 (Suppl. 3), S20-S27 (2011).

66 Burris HA 3rd, Rugo HS, Vukelja SJ et al. Phase II study of the antibody drug conjugate trastuzumab-DM1 for the treatment of human epidermal growth factor receptor 2 (HER2)-positive breast cancer after prior HER2-directed therapy. J. Clin. Oncol. 29(4), 398-405 (2011). 
67 Hurvitz S, Dirix L, Kocsis J et al. Trastuzumab emtansine (T-DM1) vs trastuzumab plus docetaxel $(\mathrm{H}+\mathrm{T})$ in previously-untreated HER2-positive metastatic breast cancer (MBC): primary results of a randomized, multicenter, open-label Phase II study (TDM4450 g/BO21976). Eur. J. Cancer 47, Abstract 5001 (2011).

68 Verma S, Dieras V, Gianni L et al. EMILIA: a Phase III, randomized, multicenter study of trastuzumab-DM1 (T-DM1) compared with lapatinib $(\mathrm{L})$ plus capecitabine $(\mathrm{X})$ in patients with HER2-positive locally advanced or metastatic breast cancer (MBC) and previously treated with a trastuzumab-based regimen. J. Clin. Oncol. 29, Abstract TPS116 (2011).

69 Mathew J, Perez EA. Trastuzumab emtansine in human epidermal growth factor receptor 2-positive breast cancer: a review. Curr. Opin Oncol. 23(6), 594-600 (2011).

70 Wong KK, Fracasso PM, Bukowski RM et al. A Phase I study with neratinib (HKI-272), an irreversible pan ErbB receptor tyrosine kinase inhibitor, in patients with solid tumors. Clin. Cancer Res. 15, 2552-2558 (2009).

71 Burstein HJ, Sun Y, Dirix LY et al. Neratinib, an irreversible ErbB receptor tyrosine kinase inhibitor, in patients with advanced ErbB2-positive breast cancer. J. Clin. Oncol. 28(8), 1301-1307 (2010).

72 Martin M, Bonneterre J, Geyer CE et al. A Phase 2, randomized, open-label study of neratinib (HKI-272) vs lapatinib plus capecitabine for 2nd/3rd-line treatment of HER 2+ locally advanced or metastatic breast cancer. Presented at: San Antonio Breast Cancer Symposium. San Antonio, TX, USA, 6-10 December 2011.

73 Andre F, Campone M, O'Regan R et al. Phase I study of everolimus plus weekly paclitaxel and trastuzumab in patients with metastatic breast cancer pretreated with trastuzumab. J. Clin. Oncol. 28, 5110-5115 (2010).

74 Dalenc F, Campone M, Hupperets P et al. Everolimus in combination with weekly paclitaxel and trastuzumab in patients (pts) with HER2-overexpressing metastatic breast cancer (MBC) with prior resistance to trastuzumab and taxanes: a multicenter Phase II clinical trial. J. Clin. Oncol. 28, Abstract 1013 (2010).

75 Morrow PK, Wulf GM, Ensor J et al. Phase I/II study of trastuzumab in combination with everolimus (RAD001) in patients with HER2-overexpressing metastatic breast cancer who progressed on trastuzumab-based therapy. J. Clin. Oncol. 29(23), 3126-3132 (2011).

76 Hickish T, Wheatley D, Lin N et al. Use of BIBW 2992, a novel irreversible EGFR/HER2 tyrosine kinase inhibitor (TKI), to treat patients with HER2-positive metastatic breast cancer after failure of treatment with trastuzumab. J. Clin. Oncol. 27(Suppl. 15), Abstract 1023 (2009).

77 Burstein HJ, Elias AD, Rugo HS et al. Phase II study of sunitinib malate, an oral multitargeted tyrosine kinase inhibitor, in patients with metastatic breast cancer previously treated with an anthracycline and a taxane. J. Clin. Oncol. 26, 1810-1816 (2008).

78 Yang SX. Bevacizumab and breast cancer: current therapeutic progress and future perspectives. Expert Rev. Anticancer Ther. 9(12), 1715-1725 (2009).

79 Gianni L, Romieu G, Lichinitser M et al. First results of AVEREL, a randomized Phase III trial to evaluate bevacizumab (BEV) in combination with trastuzumab $(\mathrm{H})$ + docetaxel (DOC) as first-line therapy for HER2-positive locally recurrent/metastatic breast cancer (LR/mBC). Presented at: San Antonio Breast Cancer Symposium. San Antonio, TX, USA, 6-10 December 2011 (Abstract S4-8).

80 Scaltriti M, Serra V, Normant E et al. Antitumor activity of the Hsp90 inhibitor IPI-504 in HER2-positive trastuzumab-resistant breast cancer. Mol. Cancer Ther. 10(5), 817-824 (2011).

\section{- Websites}

101 An Open-Label Study of Trastuzumab Emtansine (T-DM1) vs Capecitabine+Lapatinib in Patients With
HER2-Positive Locally Advanced or Metastatic Breast Cancer (EMILIA). http://clinicaltrials.gov/ct2/show/ NCT00829166

102 A Study of Trastuzumab Emtansine (T-DM1) Plus Pertuzumab/Pertuzumab Placebo Versus Trastuzumab [Herceptin] Plus a Taxane in Patients With Metastatic Breast Cancer (MARIANNE).

http://clinicaltrials.gov/ct2/show/ NCT01120184

103 A Study of Trastuzumab Emtansine in Comparison With Treatment of Physician's Choice in Patients With HER2-Positive Breast Cancer Who Have Received at Least Two Prior Regimens of HER2-Directed Therapy (TH3RESA). http://clinicaltrials.gov/ct2/show/ NCT01419197

104 Study Evaluating Neratinib Plus Paclitaxel VS Trastuzumab Plus Paclitaxel In ErbB-2 Positive Advanced Breast Cancer (NEFERTT).

http://clinicaltrials.gov/ct2/show/ NCT00915018

105 Everolimus in Combination With Trastuzumab and Paclitaxel in the Treatment of HER2 Positive Locally Advanced or Metastatic Breast Cancer (BOLERO 1). http://clinicaltrials.gov/ct2/show/ NCT00876395

106 Daily Everolimus in Combination With Trastuzumab and Vinorelbine in HER2/Neu Positive Women With Locally Advanced or Metastatic Breast Cancer (BOLERO-3). http://clinicaltrials.gov/ct2/show/ NCT01007942

107 LUX-Breast 1: BIBW 2992 (Afatinib) in HER2-positive Metastatic Breast Cancer Patients After One Prior Herceptin Treatment. http://clinicaltrials.gov/ct2/show/ NCT01125566

108 A Study of Avastin (Bevacizumab) in Combination With Herceptin (Trastuzumab)/Docetaxel in Patients With HER2 Positive Metastatic Breast Cancer. http://clinicaltrials.gov/ct2/show/ NCT00391092 